\title{
Association of Non-Alcoholic Fatty Liver Disease and Type 2 Diabetes Mellitus- An Analytical Cross-Sectional Study
}

\author{
Prateek Kaushal ${ }^{1}$, Rahul Purbey², Deepak Jain ${ }^{3}$, Kshitiz D. Vashishta ${ }^{4}$, Rajinder Singh Gupta ${ }^{5}$ \\ ${ }^{1}$ Department of General Medicine, MMIMSR, Mullana, Ambala, Haryana, India. ${ }^{2}$ Department of General \\ Medicine, MMIMSR, Mullana, Ambala, Haryana, India. ${ }^{3}$ Department of General Medicine, MMIMSR, Mullana, \\ Ambala, Haryana, India. ${ }^{4}$ Department of General Medicine, MMIMSR, Mullana, Ambala, Haryana, India. \\ ${ }^{5}$ Department of General Medicine, MMIMSR, Mullana, Ambala, Haryana, India.
}

\section{ABSTRACT}

\section{BACKGROUND}

Nonalcoholic fatty liver disease (NAFLD) is a common liver disorder but is often ignored especially in diabetes mellitus and thus carries serious complications in the long run. We aim to study the association of NAFLD with type 2 diabetes mellitus.

\section{METHODS}

A prospective observational case control study of diabetic patients was done in a tertiary care teaching hospital. A total of 50 diabetic patients fulfilling the inclusion criteria were selected as cases while 50 age sex matched non-diabetic patients were included as controls. Complete clinical, laboratory and radiological data was collected from each patient and analyzed. Data was analyzed using statistical software SPSS ver. 21.

\section{RESULTS}

Mean value of age in cases was $52.42 \pm 10.24$ years and in control was $54.04 \pm 10.16$ years ( $p>0.05$ ). There were $56 \%$ males and $44 \%$ females in both the groups. Among the cases, majority (84\%) of the patients were on Oral Hypoglycemic Agents (OHA). As compared to controls, cases had significantly higher SGOT and SGPT but significantly lower total bilirubin levels $(\mathrm{p}<0.05)$. In the lipid profile, cases had significantly higher TG, LDL and VLDL levels $(\mathrm{p}<0.05)$. Significant difference was seen in the USG findings between cases and controls. Majority (66\%) of the patients in cases had abnormal USG findings showing liver thickness, as compared to $30 \%$ in controls.

\section{CONCLUSIONS}

Type 2 diabetes was strongly found to be associated with development of NAFLD, and since most patients of NAFLD remain asymptomatic, all diabetic patients must be screened for development NAFLD.

\section{KEY WORDS}

Diabetes Mellitus, Nonalcoholic, Fatty Liver Disease
Corresponding Author:

Prateek Kaushal,

Boys Hostel,

MMIMSR, Mullana

Ambala, Haryana, India.

E-mail:dr_prateekkaushal@yahoo.com

DOI: $10.14260 / \mathrm{jemds} / 2020 / 271$

Financial or Other Competing Interests: None.

How to Cite This Article:

Kaushal P, Purbey $R$, Jain $D$, et al. Association of non-alcoholic fatty liver disease and type 2 diabetes mellitus- an analytical cross sectional study. J. Evolution Med. Dent. Sci. 2020;9(15): 1247-1251, DOI:
Submission 04-02-2020,

Peer Review 20-03-2020,

Acceptance 27-03-2020,

Published 13-04-2020. 


\section{BACKGROUND}

Diabetes and nonalcoholic fatty liver disease (NAFLD) are among the commonest health problems. The prevalence of NAFLD has been determined to 20-30 world wide ${ }^{[1]}$ and $8.7-$ $32 \%$ in India.[2] NAFLD is a condition characterized by excessive fat on liver $>5 \%$ of liver weight or $>5 \%$ of hepatocytes are affected) which occurs without any associated alcohol intake. It has a widespread histological spectrum which starts from steatosis and ranges up to steatohepatitis (NASH). Steatosis is excessive fat deposition in hepatocytes with a suggested lower threshold of at least 5 percent of hepatocytes affected and it ranges up to steatohepatitis, advanced fibrosis and rarely may even result in cirrhosis.[3] According to recent studies, development of insulin resistance or complete insulin deficiency, proinflammatory cytokine, adipokines, oxidative stress and mitochondrial dysfunction leads to initiation and progression of NAFLD. [4] The understanding about the pathology and mechanism of occurrence of NAFLD can help better identification of patients who are at risk for the disease and thus needs treatment. Management of NAFLD should start with screening and managing metabolic risk factors; thus we may be able to modify certain risk factors leading to decrease in the disease. The primary management encompasses lifestyle modifications with exercise and weight reduction, which also improves insulin sensitivity.

However, because of poor compliance in the long-term drugs aiming at reducing the insulin sensitivity levels or protecting liver from further continuing insults are often required. NAFLD is often an ignored complication of diabetes mellitus and carries serious complications in long run. The prevalence of deranged liver enzymes associated with diabetes ranges from $7.2 \%$ to $22.9 \%$, however the prevalence of NAFLD is largely not known because most the patients remain asymptomatic and it's not picked up by normally done procedures. Studies done over recent years have shown that presence of NAFLD predicts the development of diabetes and vice versa, such that each condition serving as a trigger factor for other.[5,6] This association can partly likely to be the result of a "common soil," in origin, however it may also be a result that diabetes interacting with NAFLD through a specific pathogenic process. ${ }^{[7]} \mathrm{n}$ this unique circumstance, the future difficulties for the specialist and clinician the same will incorporate expanding familiarity with the relationship among diabetes and NAFLD, a better understanding of the pathogenic variables connecting these conditions, screening for and detecting NAFLD progression noninvasively and cost viably in those in danger, and building up powerful intercessions for NAFLD in diabetes to prevent both hepatic and systemic sequel. We wanted to study the association of NAFLD with Type 2 diabetes mellitus.

\section{METHODS}

An analytical cross-sectional study was carried out in the department of Medicine of a tertiary care teaching hospital where a total of 50 diabetic cases were compared against age and gender matched 50 non-diabetic controls. The sample size was based on the study of Hardik Patel, et al[6] who observed that prevalence of NAFLD in diabetic patients was $64 \%$.

Taking this value as reference and assuming difference of $30 \%$ in prevalence rate of NAFLD between diabetic and nondiabetic patients, the minimum required sample size with $80 \%$ power of study and $5 \%$ level of significance is 40 patients in each study group. To reduce margin of error, total sample size taken is 100 (50 patients per group). A written and informed consent was obtained from all the cases and controls fulfilling the selection criteria. A detailed history was taken, and examination done was done for all the patients.

The diagnosis of Diabetes was made as per the ADA guidelines 2015 i.e., Fasting Plasma Glucose (FPG) more than or equal to $126 \mathrm{mg} / \mathrm{dl}$ (7 mmol/l), OR 2-Hours Plasma Glucose more than or equal to $200 \mathrm{mg} / \mathrm{dl}$ (11.1 mmol/l) during an Oral Glucose Tolerance test OR HbA1c more than $6.5 \%$. Patients with history of alcohol consumption, jaundice, viral hepatitis, HIV or use of hepato-toxic drugs were excluded from the study. USG was used to diagnose NAFLD. USG was able to determine any fat deposition in the Liver and negate any other cause of Liver disease. The control group included patients with no history of liver disease, no diabetes, normal liver image in ultrasound examination and normal liver laboratory tests.

Blood samples were collected after 8 hours fasting. $5 \mathrm{ml}$ of EDTA sample was collected for estimating HbA1c and $5 \mathrm{ml}$ of sample was collected in the red topped tube for getting the serum to estimate bilirubin levels, total protein and the subsets (albumin and globulin), Liver enzymes such as SGOT, SGPT, and alkaline phosphate; Lipid profile comprising of total cholesterol, triglyceride levels, high-density lipoprotein cholesterol (HDLC), low density lipoprotein cholesterol (LDLC) and very low density lipoprotein cholesterol (VLDLC) levels; and $3 \mathrm{ml}$ of blood was collected in the Sodium fluoride Grey topped vial for Fasting plasma glucose (FPG), and post prandial plasma glucose (PPPG) estimation. The estimation of the parameters was done in the fully automated Biochemistry analyser Mini VIDAS (Biomeriux instrument).

\section{Statistical Analysis}

The data collected was entered into Microsoft excel worksheet and analyzed with help of appropriate statistical methods using SPSS version 20. Categorical data was expressed as proportions and analyzed using Pearson's Chi Square Test. Continuous data was expressed as mean and standard deviation and analysed using unpaired Students T Test. $p$ value $<0.05$ was considered significant.

\section{RESULTS}

Mean value of age in cases was $52.42 \pm 10.24$ years and in control was $54.04 \pm 10.16$ years. ( $>0.05$ ) There were $56 \%$ males and $44 \%$ females in both the groups. Among the cases, majority (84\%) of the patients were on Oral hypoglycaemic 
agents (OHA). The anthropometry was comparable among the cases and controls. (table 1).

As compared to controls, cases had significantly higher SGOT and SGPT but significantly lower total bilirubin levels. $(p<0.05)$ Rest of the biochemical parameters were comparable as shown in the Table 2. As compared to controls, cases had significantly higher TG, LDL and VLDL levels. $(p<0.05)$ Rest of the lipid profile parameter were comparable among cases and controls as shown in Table 3. Significant difference was seen in the USG findings between cases and control. Majority (66\%) of the patients in cases had abnormal USG findings showing Liver thickness, as compared to $30 \%$ in control. (table 4).

\section{DISCUSSION}

In this study, out of 50 patients, prevalence of NAFLD as evident from increased echogenicity, was found in 33 patients i.e. $66 \%$ of the patients whereas in the control group, NAFLD was present in only $15(30 \%)$ of the patients. The difference between the two groups is statistically significant with a $\mathrm{p}$ value of 0.0003 . The overall prevalence of NAFLD in India is 8.7 to $32 \%{ }^{[2]}$ Similar results were reported in a Japanese study conducted by Jimba S. et al,[8] in which there increase in the prevalence of NAFLD in patients having impaired fasting glucose and in patients having type 2 diabetes mellitus was $43 \%$ and $62 \%$, respectively. The age involvement for diabetes and NAFLD in our study was in the fifties as seen in other studies. ${ }^{[7,8]}$ The elderly age is common for the occurrence of the diabetes and the continuing changes in the Liver predispose an individual to fatty changes.

In recent times, rising prevalence rates of the NAFLD's risk factors have been as follows: obesity, diabetes mellitus (DM), and metabolic syndrome. The concomitant occurrence of these co-morbidities increases the chances of NAFLD in diabetics and subsequently also increase the chances of Cardiovascular disease and mortality. In our study, we found a significant association of liver enzymes derangement (SGOT, SGPT) and lipid profile derangements (TG, LDL, VLDL) among the diabetics as compared to the controls.

\begin{tabular}{|c|c|c|c|c|}
\hline \multirow{2}{*}{$\begin{array}{c}\text { Anthropometric } \\
\text { Parameters }\end{array}$} & \multicolumn{2}{|c|}{ Group } & \multirow{2}{*}{ Total } & \multirow[b]{2}{*}{$\mathbf{p}$} \\
\hline & Case $(n=50)$ & Control $(n=50)$ & & \\
\hline \multicolumn{5}{|c|}{ Height (in meters) } \\
\hline Mean \pm S.D. & $1.67 \pm 0.08$ & $1.68 \pm 0.1$ & $1.68 \pm 0.09$ & \\
\hline Median(IQR) & $1.67(1.600-1.760)$ & $1.7(1.580-1.780)$ & $1.68(1.590-1.765)$ & \\
\hline \multicolumn{5}{|c|}{ Weight (in kg) } \\
\hline Mean \pm S.D. & $76.5 \pm 9.83$ & $77.38 \pm 10.89$ & $76.94 \pm 10.33$ & \\
\hline Median(IQR) & $76(69-84)$ & $76(68-86)$ & $76(68.500-85)$ & 0.674 \\
\hline \multicolumn{5}{|c|}{ Body Mass Index $\left(\mathrm{Kg} / \mathrm{m}^{2}\right)$} \\
\hline Normal & $2(4.00 \%)$ & $2(4.00 \%)$ & $4(4.00 \%)$ & \\
\hline Obesity & $43(86.00 \%)$ & $43(86.00 \%)$ & $86(86.00 \%)$ & 1.000 \\
\hline Severe obesity & $5(10.00 \%)$ & $5(10.00 \%)$ & $10(10.00 \%)$ & \\
\hline Mean \pm S.D. & $27.31 \pm 1.8$ & $27.38 \pm 1.66$ & $27.35 \pm 1.72$ & \\
\hline Median(IQR) & $26.94(26.11-28.30)$ & $27.2(26.100-28.300)$ & $\begin{array}{c}27.19(26.100- \\
28.300)\end{array}$ & 0.832 \\
\hline
\end{tabular}

For the first time, the causal association between NAFLD and type 2 diabetes mellitus (T2DM) was found when high levels of alanine aminotransferase (ALT) were noticed to estimate T2DM development in Pima Indians. ${ }^{[9]}$ To date, it has been reported by several prospective, population-based cohort studies that increased levels of serum liver enzymes could elevate the risk of T2DM, which is independent of common risk factors, e.g. diet and lifestyle, among individuals belonging to different ethnic backgrounds.[3] Such studies are, however, limited in number as most of the patients with NAFLD have normal levels of transaminase and complex explanations for the elevation of the liver enzyme.

\begin{tabular}{|c|c|c|c|c|}
\hline \multirow{2}{*}{$\begin{array}{l}\text { Biochemical } \\
\text { Parameters }\end{array}$} & \multicolumn{2}{|c|}{ Group } & \multirow{2}{*}{ Total } & \multirow[b]{2}{*}{$\mathbf{p}$} \\
\hline & Case $(n=50)$ & Control $(n=50)$ & & \\
\hline \multicolumn{5}{|c|}{ Total bilirubin(mg/dl) } \\
\hline $\begin{array}{l}\text { Deranged } \\
\text { Normal }\end{array}$ & $\begin{array}{c}3(6.00 \%) \\
47(94.00 \%)\end{array}$ & $\begin{array}{c}4(8.00 \%) \\
46(92.00 \%)\end{array}$ & $\begin{array}{c}7(7.00 \%) \\
93(93.00 \%)\end{array}$ & 1.000 \\
\hline $\begin{array}{c}\text { Mean } \pm \text { S.D. } \\
\text { Median (IQR) }\end{array}$ & $\begin{array}{c}0.46 \pm 0.3 \\
0.34(0.24-0.63)\end{array}$ & $\begin{array}{c}0.66 \pm 0.28 \\
0.74(0.420-0.870)\end{array}$ & $\begin{array}{c}0.56 \pm 0.31 \\
0.48(0.300-0.785)\end{array}$ & 0 \\
\hline \multicolumn{5}{|c|}{ Direct bilirubin(mg/dl) } \\
\hline $\begin{array}{l}\text { Deranged } \\
\text { Normal }\end{array}$ & $\begin{array}{l}39(78.00 \%) \\
11(22.00 \%)\end{array}$ & $\begin{array}{l}35(70.00 \%) \\
15(30.00 \%)\end{array}$ & $\begin{array}{l}74(7 \\
26(2\end{array}$ & 0.362 \\
\hline $\begin{array}{l}\text { Mean } \pm \text { S.D. } \\
\text { Median (IQR) }\end{array}$ & $\begin{array}{c}0.22 \pm 0.18 \\
0.15(0.12-0.20)\end{array}$ & $\begin{array}{c}0.26 \pm 0.19 \\
0.18(0.140-0.320)\end{array}$ & $\begin{array}{r}0.24 \\
0.17(0.13\end{array}$ & 0.068 \\
\hline \multicolumn{5}{|c|}{ SGOT(u/l) } \\
\hline $\begin{array}{c}\text { Deranged } \\
\text { Normal }\end{array}$ & & & $\begin{array}{l}\%) \\
\%)\end{array}$ & 0.027 \\
\hline Mean \pm S.D. & $39.82 \pm 14.02$ & 34.4 & $37.12 \pm$ & \\
\hline Median ( & & $35(28-3$ & & .0 \\
\hline \multicolumn{5}{|c|}{ SGPT(u/l) } \\
\hline Deranged & & $27(54.00 \%)$ & $67(67$ & 0.006 \\
\hline $\begin{array}{l}\text { Mean } \pm \text { S.D. } \\
\text { Median (IQR) }\end{array}$ & $\begin{array}{l}46.18 \pm 14.55 \\
47.5(39-55)\end{array}$ & $\begin{array}{l}38.62 \pm 12.67 \\
38.5(29-51)\end{array}$ & $\begin{array}{c}42.4 \pm 14.1 \\
43.5(31-54)\end{array}$ & 0.007 \\
\hline \multicolumn{5}{|c|}{ Alkaline phosphatase (u/l) } \\
\hline $\begin{array}{l}\text { Deranged } \\
\text { Normal }\end{array}$ & $\begin{array}{l}18(36.00 \%) \\
32(64.00 \%)\end{array}$ & $\begin{array}{l}22(44.00 \%) \\
28(56.00 \%)\end{array}$ & $\begin{array}{l}40(40.00 \%) \\
60(60.00 \%)\end{array}$ & 0.414 \\
\hline $\begin{array}{l}\text { Mean } \pm \text { S.D. } \\
\text { Median (IQR) }\end{array}$ & $\begin{array}{c}112.66 \pm 36.1 \\
109.5(94-122)\end{array}$ & $\begin{array}{c}105.08 \pm 27.52 \\
105.5(82-126)\end{array}$ & $\begin{array}{l}108.87 \pm 32.16 \\
109(89-125)\end{array}$ & 0.501 \\
\hline \multicolumn{5}{|c|}{ Albumin(gm/dl) } \\
\hline $\begin{array}{c}\text { Deranged } \\
\text { Normal }\end{array}$ & $\begin{array}{l}31(62.00 \%) \\
19(38.00 \%)\end{array}$ & $\begin{array}{l}27(54.00 \%) \\
23(46.00 \%)\end{array}$ & $\begin{array}{l}58(58.00 \%) \\
42(42.00 \%)\end{array}$ & 0.418 \\
\hline Mean \pm S.D. & $3.37 \pm 0.63$ & $3.56 \pm 0.58$ & $3.46 \pm 0.61$ & 0.166 \\
\hline Median (IQR) & $3.3(3-3.700)$ & $3.4(3.100-4)$ & $3.35(3.100-3.900)$ & 0.166 \\
\hline \multicolumn{5}{|c|}{ ble 2. Comparison of Biochemical Parameters } \\
\hline
\end{tabular}

\begin{tabular}{|c|c|c|c|c|}
\hline \multirow{2}{*}{$\begin{array}{l}\text { Lipid Profile } \\
\text { Parameters }\end{array}$} & \multicolumn{2}{|c|}{ Group } & \multirow{2}{*}{ Total } & \multirow{2}{*}{$\mathbf{p}$} \\
\hline & Case $(n=50)$ & Control $(n=50)$ & & \\
\hline \multicolumn{5}{|c|}{ Cholesterol(mg/dl) } \\
\hline Deranged & $17(34.00 \%)$ & $15(30.00 \%)$ & $32(32.00 \%)$ & \multirow{2}{*}{0.668} \\
\hline Normal & $33(66.00 \%)$ & $35(70.00 \%)$ & $68(68.00 \%)$ & \\
\hline Mean \pm S.D. & $179.7 \pm 32.21$ & $174.38 \pm 39.23$ & $177.04 \pm 35.81$ & \multirow{2}{*}{0.46} \\
\hline Median (IQR) & $177.5(156-212)$ & $172.5(144-206)$ & $177(154$ & \\
\hline \multicolumn{5}{|c|}{ Triglycerides $(\mathrm{mg} / \mathrm{dl})$} \\
\hline Deranged & $23(46.00 \%)$ & $12(24.00 \%)$ & $35(35.00 \%)$ & \multirow{2}{*}{0.021} \\
\hline Normal & $27(54.00 \%)$ & $38(76.00 \%)$ & $65(65.00 \%)$ & \\
\hline Mean \pm S.D. & $168.24 \pm 60.9$ & $126.8 \pm 42.69$ & $147.52 \pm 56.31$ & \multirow{2}{*}{0.0001} \\
\hline Median(IQR) & $159(126-190)$ & $118.5(89-162)$ & $141(100-176)$ & \\
\hline \multicolumn{5}{|c|}{ High density lipoprotein(mg/dl) } \\
\hline Deranged & $5(10.00 \%)$ & $2(4.00 \%)$ & $7(7.00 \%)$ & \multirow{2}{*}{0.436} \\
\hline Normal & $45(90.00 \%)$ & $48(96.00 \%)$ & $93(93.00 \%)$ & \\
\hline Mean \pm S.D. & $38.28 \pm 7.66$ & $38.94 \pm 5.88$ & $38.61 \pm 6.8$ & \multirow{2}{*}{0.63} \\
\hline Median(IQR) & $38(34-43)$ & $40(34-42)$ & $39(34-42)$ & \\
\hline \multicolumn{5}{|c|}{ Low density lipoprotein(mg/dl) } \\
\hline Deranged & $1(2.00 \%)$ & $0(0.00 \%)$ & $1(1.00 \%)$ & \multirow{2}{*}{1.000} \\
\hline Normal & 49 (98.00\%) & $50(100.00 \%)$ & $99(99.00 \%)$ & \\
\hline Mean \pm S.D. & $120.58 \pm 27.47$ & $99.74 \pm 24.88$ & $110.16 \pm 28.1$ & \multirow{2}{*}{0.0001} \\
\hline Median(IQR) & $118(98-138)$ & $90(87-110)$ & $105(89-132)$ & \\
\hline \multicolumn{5}{|c|}{ Very low-density lipoprotein(mg/dl) } \\
\hline Deranged & $17(34.00 \%)$ & $2(4.00 \%)$ & $19(19.00 \%)$ & \multirow{2}{*}{0.0002} \\
\hline Normal & $33(66.00 \%)$ & $48(96.00 \%)$ & $81(81.00 \%)$ & \\
\hline Mean \pm S.D. & $32.79 \pm 11.69$ & $24.55 \pm 7.93$ & $28.67 \pm 10.77$ & \multirow{2}{*}{$<.0001$} \\
\hline Median(IQR) & $30.7(26-37.20)$ & $23.6(19-30)$ & $28(21-34)$ & \\
\hline Tabl & Comparison o & Lipid Profile & arameters & \\
\hline
\end{tabular}

\begin{tabular}{|ccccc|}
\hline USG & \multicolumn{2}{c|}{ Group } & \multirow{2}{*}{ Total } & p value \\
Findings & Case $(\mathbf{n = 5 0 )}$ & Control $(\mathbf{n = 5 0})$ & & \\
No & $17(34.00 \%)$ & $35(70.00 \%)$ & $52(52.00 \%)$ & \\
Yes & $33(66.00 \%)$ & $15(30.00 \%)$ & $48(48.00 \%)$ & 0.0003 \\
Total & $50(100.00 \%)$ & $50(100.00 \%)$ & $100(100.00 \%)$ \\
\hline \multicolumn{5}{|c|}{ Table 4. Comparison of USG Findings } \\
\hline
\end{tabular}

In another study by Kempinski R et al (2019)[10] it was shown that overweight, obesity, and metabolic syndrome along with its components (i.e., hypertension, T2DM, dyslipidemia) were more common in patients with NAFLD than matched controls. The authors also found that patients with NAFLD are usually affected by gastric disorders, colonic diverticulosis, and cholecystolithiasis. This further stresses 
on the fact that such patients are prone to other GIT diseases also because of the diabetic effects on the autonomic nervous system causing nerve degeneration. Such comorbidities negatively affect the natural course of NAFLD. In NAFLD patients, presence of T2DM and obesity greatly influence the progression to fibrosis. In a very recent review, shared gene/protein interaction networks were deciphered, and gene-disease analysis was performed. In the review, shared mechanisms between the NAFLD and the MetS diseases were also reported. This review also Shared mechanisms among NAFLD and the MetS diseases were revealed and presented evidence that NAFLD, particularly NASH, needs taking multitarget approaches, instead of concentrating on single mechanisms related to disease.[11] Definitely, in the present study, the strong connections between the insulin resistance and the NAFLD was indirectly confirmed.

In our study, the ultrasonography (USG) findings also showed increased NAFLD among diabetics as compared to non-diabetics; thus, laying down the complex process of occurrence of NAFLD in relation to the metabolic derangements. Studies have demonstrated that in different populations, NAFLD that is diagnosed by USG is found to be associated with a 33\% to a fivefold increased risk of T2DM with several follow-up periods as well as severity of NAFLD. Advancement in non-invasive methods for assessment of liver fat content have allowed studies on the quantitative relationship between the NAFLD and the T2DM over the last decade. Based on the liver fat content measured by proton magnetic resonance spectroscopy (1H-MRS), A clear threshold of the liver fat content of nearly $6 \pm 2 \%$ was observed, on the basis of liver fat content that was measured by proton magnetic resonance spectroscopy (1H-MRS), following which metabolic disorders (e.g., hypertriglyceridemia, hypo-high-density lipoprotein cholesterolaemia, muscle insulin resistance) was completely established. ${ }^{[3]}$ As reported by the Shanghai Changfeng Community study, the liver fat content more than $10 \%$ (assessed by a quantitative ultrasound method) was found to be associated with increase in systemic insulin resistance as well as diabetes.[12] When these results were considered together, the significance of NAFLD in the occurrence of diabetes was highlighted.

NAFLD and T2DM association is both ways: (1) in diabetes, NAFLD increases and (2) T2DM is also a strong clinical predictor of the NAFLD'S progression to cirrhosis as well as NASH. Present-day estimates suggest that approximately $10-20 \%$ of patients with NAFLD will progress into non-alcoholic steatohepatitis (NASH), therefore raising the risk of cirrhosis or advanced fibrosis in the liver. The existence of T2DM raises NASH's risk by two- to threefold.[13] Explanation for this is that in T2DM patients, there is increased liver lipogenesis and fatty acid oxidation and triglyceride secretion through very low-density lipoprotein (VLDL) is reduced. Furthermore, fatty acid release from adipose tissue is increased by the peripheral insulin resistance. Moreover, peripheral insulin resistance increases fatty acid release from adipose tissue and the hepatic uptake of fatty acids under the insulin resistance status is also upregulated. Therefore, there is usually co-existence of NAFLD with T2DM. Originally, the mild accumulation of liver fat is an adaptive response to metabolic stress against the lipotoxicity of free fatty acids. But, on the background of continuous influx of hepatic free fatty acid, there is activation of several inflammatory pathways with the increase in the hepatic intracellular triglycerides. Recently genetic variants have been studied which are more linked to the development of these condition together; which however was not done in the study.[3]

We in our study included only USG findings for the diagnosis of NAFLD which in itself is a methodological limitation of the study as liver biopsy is still considered to be a gold standard technique for the diagnosis of NAFLD as well as its stage, that is simple steatosis, steatohepatitis, fibrosis, and cirrhosis. Therefore, since it is an invasive procedure that usually involves hospital admission, non-invasive methods are used, particularly to recognize patients with liver fibrosis. For diagnosing liver steatosis, abdominal USG is a simple method; however, like computed tomography (CT) or magnetic resonance imaging (MRI), it does not accurately define fibrosis in the liver. Transient elastography measures stiffness in liver tissue in USG method; it is a new imaging technique for assessing patients having liver fibrosis. Serum cytokeratin-18 is considered to be a promising as well as an accurate non-invasive marker of NASH amongst the novel diagnostic techniques. Staging of liver fibrosis with simple serum marker panels remains a challenge, with FIB-4 test and NAFLD fibrosis score being the most accurate. The 13Cmethacetin breath test is the new method for detection of fibrosis in NAFLD.[3]

\section{Limitations}

The main limitation of the study was the small sample size. Thus, we recommend large multicentric studies to understand the intricate relationship of diabetes and NAFLD. Secondly, long term follow-up of the patients were not done and other complications and associations like cardiovascular diseases and hypertension were not assessed. Lastly, fibroscan was not done in our study which could have helped assess the severity of fibrosis in such patients.

\section{CONCLUSIONS}

NAFLD is linked with diabetes due to a common path of occurrence involving obesity and metabolic derangements and thus liver must be routinely screened in diabetics to prevent long term complications.

\section{REFERENCES}

[1] Bedogni G, Miglioli L, Masutti F, et al. Prevalence of and risk factors for nonalcoholic fatty liver disease: the Dionysos nutrition and liver study. Hepatology 2005;42(1):44-52.

[2] Das K, Das K, Mukherjee PS, et al. Non-obese population in a developing country has a high prevalence of nonalcoholic fatty liver and significant liver disease. Hepatology 2010;51(5):1593-602. 
[3] Xia MF, Bian H, Gao X. NAFLD and diabetes: two sides of the same coin? Rationale for gene-based personalized NAFLD treatment. Front Pharmacol 2019;10:877.

[4] Farrell GC, Larter CZ. Nonalcoholic fatty liver disease: from steatosis to cirrhosis. Hepatology 2006;43(2 Suppl 1):S99-S112.

[5] Musso G, Gambino R, Cassader M, et al. Meta-analysis: natural history of Non-Alcoholic Fatty Liver Disease (NAFLD) and diagnostic accuracy of non-invasive tests for liver disease severity. Ann Med 2011;43(8):617-49.

[6] Patel H, Verma YN. Prevalence of non-alcoholic fatty liver disease in type-2 diabetes mellitus patients. Int J Res Med Sci 2018;6(4):1322-6.

[7] Lo L, McLennan SV, Williams PF, et al. Diabetes is a progression factor for hepatic fibrosis in a high fat fed mouse obesity model of non-alcoholic steatohepatitis. J Hepatol 2011;55(2):435-44.

[8] Jimba S, Nakagami T, Takahashi M, et al. Prevalence of non-alcoholic fatty liver disease and its association with impaired glucose metabolism in Japanese adults. Diabet Med 2005;22(9):1141-5.
[9] Vozarova B, Stefan N, Lindsay RS, et al. High alanine aminotransferase is associated with decreased hepatic insulin sensitivity and predicts the development of type 2 diabetes. Diabetes 2002;51(6):1889-95.

[10] Kempiński R, Łukawska A, Krzyżanowski F, et al. Clinical outcomes of non-alcoholic fatty liver disease: polish-case control study. Adv Clin Exp Med 2019;28(12):1615-20.

[11] Sookoian S, Pirola CJ. Review article: Shared disease mechanisms between non-alcoholic fatty liver disease and metabolic syndrome - translating knowledge from systems biology to the bedside. Aliment Pharmacol Ther 2019;49(5):516-27.

[12] Xia MF, Bian H, Yan HM, et al. Assessment of liver fat content using quantitative ultrasonography to evaluate risks for metabolic diseases. Obesity (Silver Spring) 2015;23(9):1929-37.

[13] Portillo-Sanchez P, Bril F, Maximos M, et al. High prevalence of nonalcoholic fatty liver disease in patients with type 2 diabetes mellitus and normal plasma aminotransferase levels. J Clin Endocrinol Metab 2015;100(6):2231-8. 